\title{
FIB / FESEM Investigations of Polymer / Inorganic Composites
}

\author{
Brian P. Gorman, Laxmi Sahu, Ali Shaito, and Nandika A. D’Souza \\ Department of Materials Science and Engineering, University of North Texas, Denton, TX, USA \\ 76203
}

Oscar Olea-Mejia, Witold Brostow

LAPOM, University of North Texas, Denton, TX, USA

Polymer - inorganic composites have many applications in materials science. Composites with inorganic particles intermixed within the polymer matrix must be characterized for dispersion both across the surface as well as through the depth of the material. Dual column focused ion beam (FIB) and field emission scanning electron microscopy (FESEM) are well suited for this type of analysis, however, there is little data in the literature regarding FIB milling of polymeric materials.

FIB milling of polymers can be more troublesome than metals, ceramics, or semiconductors. As seen in Figure 1, room temperature FIB milling of low glass transition temperature $\left(\mathrm{Tg} \sim-100^{\circ} \mathrm{C}\right)$ polymers can result in mechanical damage and destruction of the sample matrix. The purpose of this study was to examine the problems with room temperature FIB milling of low-Tg polymers and to remedy these issues by changing the FIB milling parameters.

Experiments in this study were conducted on several polymeric materials with different $\mathrm{Tg}$ in order to determine the effects of sample heating due to FIB milling (FEI Nova 200). Our experiments have illustrated that a change in ion beam current drastically changes the amount of damage in the polymer. As seen in the SRIM [2] simulations in figure 2, the range of $30 \mathrm{keV} \mathrm{Ga}$ ions in polypropylene (a polymer with similar composition and density to those used in this study) is approximately $80 \mathrm{~nm}$ parallel to the beam and $50 \mathrm{~nm}$ laterally. In order to reduce the effects of beam heating, the milling parameters were set up such that the overlap between the subsequent milling spots were within the outer ranges of the beam straggle. For example, a 30nm beam spot size mill was set up such that subsequent milling spots were approximately $75 \mathrm{~nm}$ apart. This reduces the collision cascade overlap, and thus reduces the localized heating of the material.

Figure 3 is an SEM micrograph of the same material in figure 1 milled using the modified beam parameters. As can be seen, the structure suffers very little beam damage, and the cross section with metal particles dispersed in it are easily recognized. Again using the same milling parameters, a polymer with a higher $\operatorname{Tg}\left(\sim 30^{\circ} \mathrm{C}\right)$ is also milled successfully (figure 4$)$, and the clay nanoparticles dispersed throughout the matrix are easily distinguished. Materials with even higher $\operatorname{Tg}\left(\sim 200^{\circ} \mathrm{C}\right)$ are also easily milled with these parameters (figure 5), and the underlying structures of the inorganics are again easily distinguished. These altered milling parameters will allow for subsequent milling, and ultimately $3-\mathrm{D}$ reconstruction of the polymer - inorganic composite structure.

References

[1] L. Giannuzzi and F. A. Stevie, eds., Introduction to Focused Ion Beams, Springer (2005), and references therein.

[2] www.srim.org 


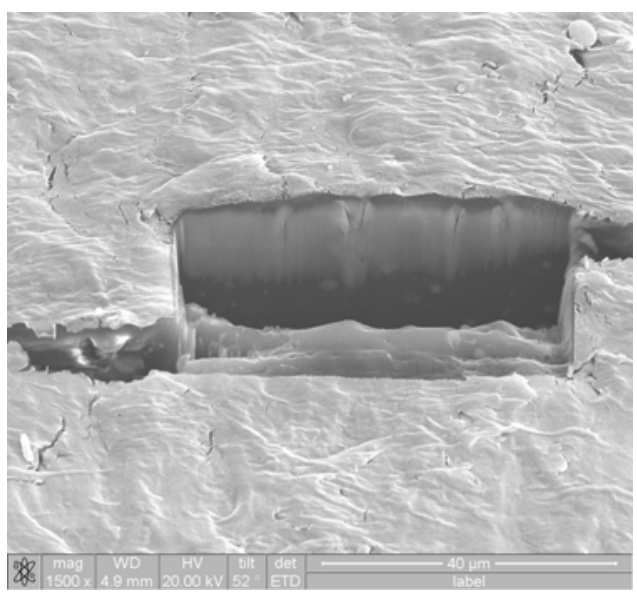

FIG. 1. SEM image of a polymer - metal particle composite illustrating cracking due to sample heating.

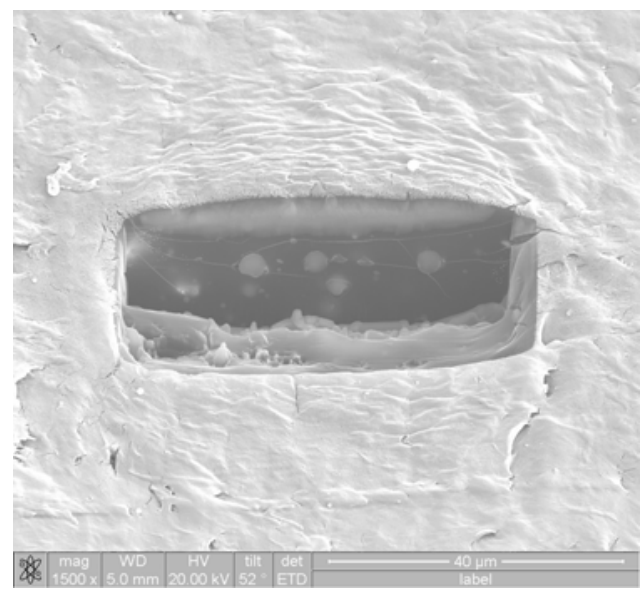

FIG. 3. SEM image of the same material in figure 1 milled using the modified FIB parameters to reduce the sample heating.

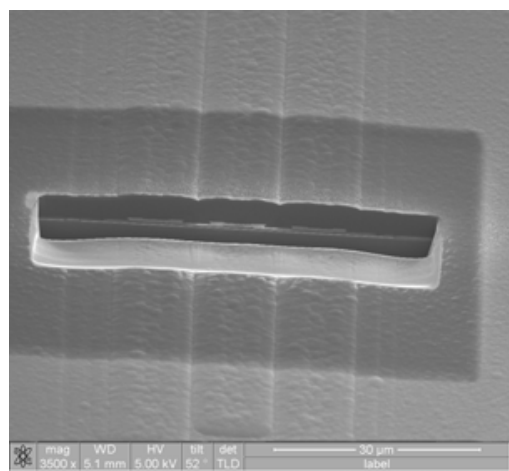

FIG. 5. SEM image of a polymer capped inorganic composite material again showing good FIB milling parameters. (a)

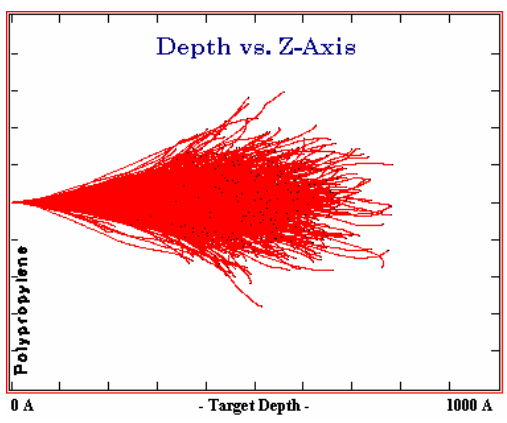

(b)

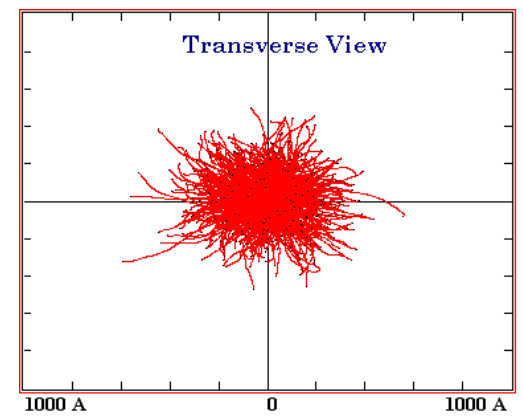

FIG. 2. SRIM simulations for the (a) depth and (b) lateral straggle of $30 \mathrm{keV} \mathrm{Ga}$ ions into polypropylene.

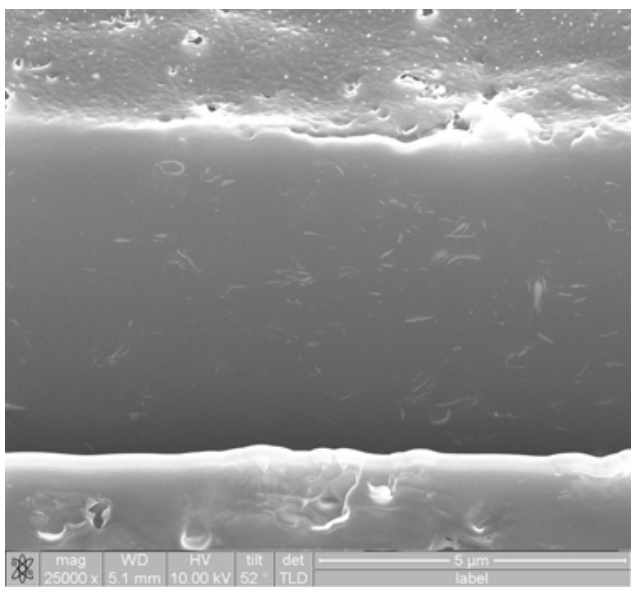

FIG. 4. SEM image of a polymer / clay nanoparticle composite FIB milled using the modified beam parameters. 